\title{
Fundamental approach on orthotic device for patients with osteoarthritis of knee considering screw home movement
}

\author{
Zhiqiang WU *, Tomonori YAMAMOTO*, Rafiuddin SYAM** and Satoru SHIBATA* \\ * Department of Mechanical Engineering, Ehime University \\ 3 Bunkyo-cho, Ehime 790-8577, Japan \\ E-mail: wu.zhiqiang.mh@ehime-u.ac.jp \\ ** Mechanical Engineering Department, Hasanuddin University \\ Makassar, Indonesia
}

Received 6 May 2016

\begin{abstract}
In this study, as a fundamental approach to realize orthotic device for patients with osteoarthritis of the knee considering screw home movement, bending and extension movements of lower thigh of able-bodied persons are analyzed and inherent screw home movement is examined. The analyzed extension movement of lower thigh with screw home movement is mathematically modeled and the structure of an orthotic device is proposed based on the model. It is confirmed by the simulation based on FEM analysis that the force acting on the leg when using the proposed orthotic device is helpful to realize normal screw home movement and valgus rotation, which is expected to be useful for patients with osteoarthritis of the knee.
\end{abstract}

Key words : Orthotic device, Osteoarthritis of the knee, Screw home movement, Valgus rotation, Mathematical model, FEM analysis

\section{Introduction}

With the rapid progress of the aging society, the number of patients with trouble in bone or arthrosis has been increased recently. There are many patients suffered from osteoarthritis of the knee. Osteoarthritis is debilitating affection in which arthritis occurs due to wear-out of cartilage with age and pain lasts while walking. The treatment options for patients with osteoarthritis include, but are not limited to, surgery, the use of wedge insoles and the use of knee braces. It was reported that the operative task to aged osteoarthritis was related to their declining general medical condition (Goldberg, 1992). The treatment using wedge insoles has been shown to be effective only in early-stage osteoarthritis (Sasaki, and Yasuda, 1987, Yasuda, and Sasaki, 1987). Knee bracing for osteoarthritis has gained popularity due to its ability to provide patients with pain relief while being relatively easy to use (Matsuno, et al., 1997, Self, et al., 2000).

For the development of knee braces, the earlier studies focused on providing pain relief by reducing the load applied to the knee during the stance phase of gait. Various kinds of brace are designed for this purpose. A study by Pollo et al. (1994) showed that wearing a valgus knee brace can reduce the varus moment during stance. Self et al. (2000) measured the force applied to the lateral side of the knee by a Monarch brace, a kind of valgus knee brace, and showed that it can reduce the varus moment at $20 \%$ and $25 \%$ at stance. Esrafilian et al. (2012) designed a knee orthosis whose rotational axes can be adjusted by external screws, and its effect on walking is evaluated experimentally.

However, in the stance phase, since the brace has to be able to provide enough force to oppose the varus moment caused by the force of body weight, the brace should be designed stiff enough. So, the brace becomes heavy and the contact force applied from the brace to the leg becomes large.

In recent years, different approaches have been proposed to avoid pain while walking by correcting knee movement and posture during the swing phase. It is known that knee joint movement of patients of osteoarthritis is different from that of able-bodied person. In the case of healthy knee, lower thigh conducts slight valgus rotation and screw home movement (SHM) when extending from bending posture. Yamasaki and Moriwaki (2005) developed a 
brace composed of gear and rack considering SHM. The gear and rack mechanism was also applied to design a soft type OA support by $\mathrm{Wu}$, et al. (2008). However, resistance to gear becomes large when extending knee, which interferes natural movement. On the other hand, a knee brace using cam mechanism was developed (Postelmans, 1997). The cam curve is designed so that natural extorsion movement can be generated synchronizing with extension movement of the knee. However, even in this mechanism, SHM is not realized well.

From these points of view, we aim at developing orthotic device which can generate slight valgus rotation and SHM. As a fundamental approach to realize it, the followings are conducted. Firstly, knee joint movement of human subjects in extending their leg from bending posture are measured. Secondly, by analyzing the obtained data, slight valgus rotation and screw home movement are modeled mathematically. Thirdly, the structure and shape of the orthotic device are proposed and determined based on the mathematical model. Finally, the effectiveness of the orthotic device is discussed through computer simulation using finite element analysis.

\section{Measurement and analysis of valgus rotation and SHM in lower thigh when extending from bending posture}

\subsection{Measurement of movement of knee joint when extending from bending posture}

To measure the movement extending his or her leg from bending posture a three dimensional motion capture system is used. In the three dimensional motion capture system, infrared radiations are projected from the surfaces of plural cameras, and the movements of the reflecting markers attached to the object are acquired by the cameras. To make the analysis of the movement of knee joint easy, number 1, 2 and 3 of reflecting marker are attached to the femur and number 4, 5 and 6 of reflecting marker are attached to the lower thigh, and number 7 is attached to the side face of the center of the knee outside of right leg as shown in Fig.1. The weight of reflecting marker is very light and does not affect human natural movement.

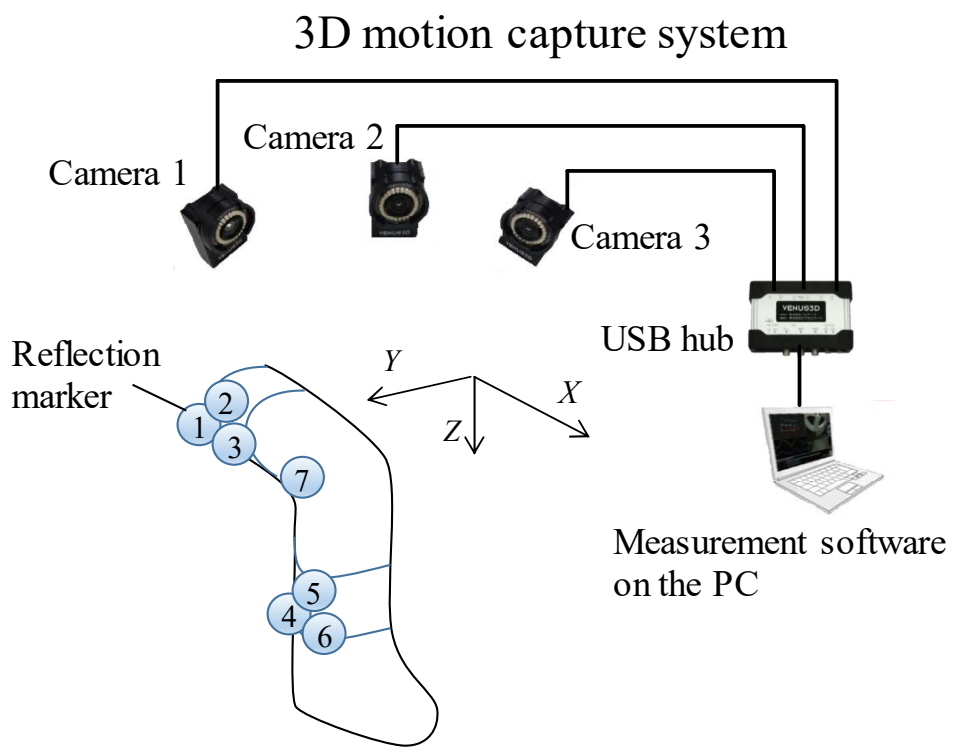

Fig.1 Positions of markers to be measured

In the experiments, ten subjects sitting on a chair repeat bending and stretching 10 times and the movements of femur and lower thigh are measured. In the experiments, $X$ axis is defined as the front direction to the knee, $Y$ axis is defined as the outer lateral direction to the knee, and $Z$ axis is defined as the vertical down direction. The measured position data of all the markers are expressed as this coordinate system.

\subsection{Analysis of the movement of lower thigh and its modeling}

In this research, it is assumed that degree-of-freedom at knee joint is composed of three rotations such as bending and stretching, valgus and varus, and SHM, and that their axes cross at the center of knee joint.

To analyze the movement of lower thigh of able-bodied person, angles $\theta_{1}, \theta_{2}$ and $\theta_{3}$ are defined to the center of the 


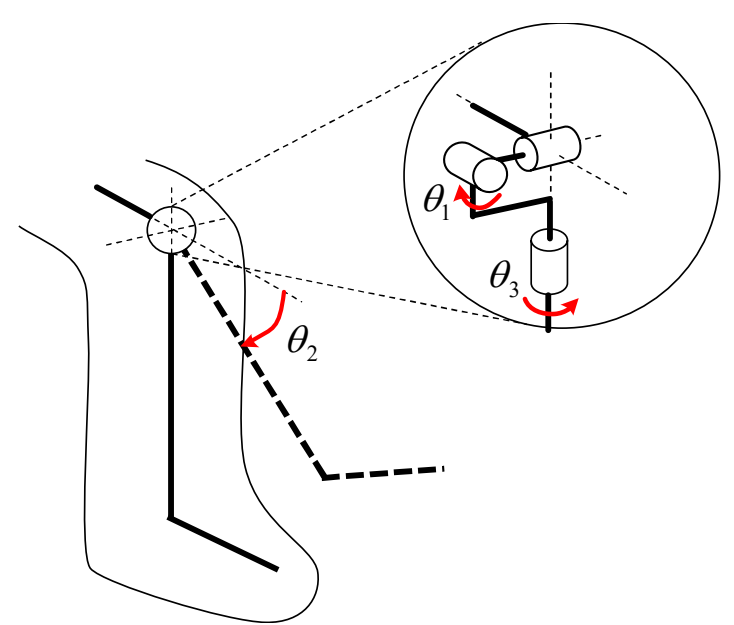

Fig.2 Definition of rotational angle of $\theta_{1}, \theta_{2}$, and $\theta_{3}$

knee as shown in Fig.2. $\theta_{1}$ is used to examine valgus rotation, $\theta_{2}$ is used to measure the rotational angle of bending and stretching, and $\theta_{3}$ is used to examine SHM. When femur and lower thigh are on the straight line (that is, extending completely), the value of $\theta_{2}$ is defined as zero, and when femur is perpendicular to lower thigh, the value of $\theta_{2}$ is defined as 90 degree. Angles $\theta_{1}, \theta_{2}$ and $\theta_{3}$ are calculated accurately by the measured values of marker 1 to marker 7 .

The average value of the change of $\theta_{1}$ to the change of $\theta_{2}$ for five subjects obtained in the experiment is shown by lozenge in Fig.3. As shown in the figure, $\theta_{1}$ decreases gently as $\theta_{2}$ increases, which shows valgus rotation of lower thigh when stretching knee from bending posture. Mathematical modelling of the experimental result using cubic polynomial is attempted. As the result, the next polynomial is obtained by the least squares method.

$$
\theta_{1}=-0.0001 \theta_{2}^{3}+0.0144 \theta_{2}{ }^{2}-0.679 \theta_{2}+20.594
$$

Red line in Fig.3 shows the approximated curve by the mathematical model. As shown in the figure, the model can reproduce human characteristics accurately.

The average value of the change of $\theta_{3}$ to the change of $\theta_{2}$ for five subjects obtained in the experiment is shown by lozenge in Fig.4. As shown in the figure, $\theta_{3}$ increases gently as $\theta_{2}$ increases, which shows SHM of lower thigh when stretching knee from bending posture. In the wave profile an inflection point can be observed during increase. So, mathematical modelling of the experimental result using cubic polynomial is attempted. As the result, the next polynomial is obtained by the least squares method.

$$
\theta_{3}=0.0002 \theta_{2}^{3}-0.0393 \theta_{2}^{2}+2.2822 \theta_{2}-62.357
$$

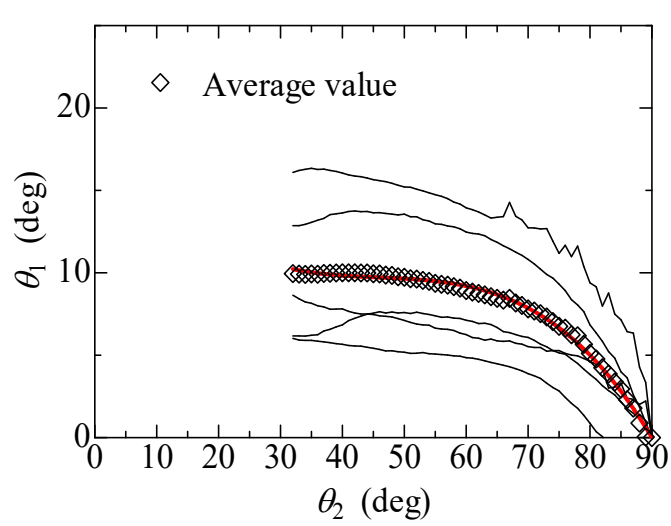

Fig.3 Relation between $\theta_{2}$ and $\theta_{1}$, and its mathematical model

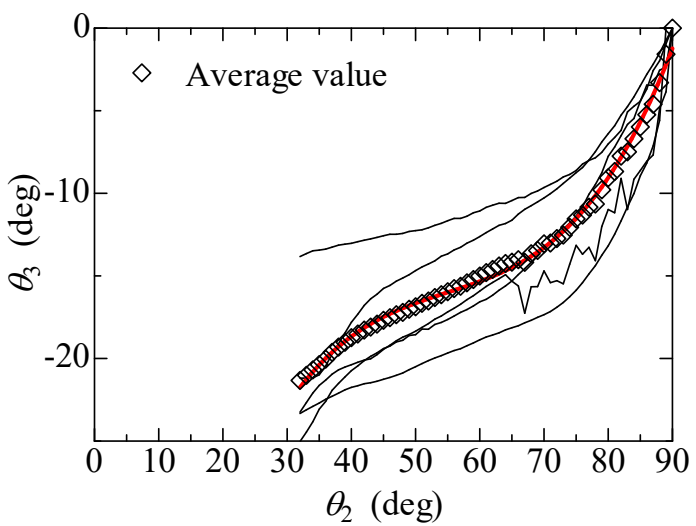

Fig.4 Relation between $\theta_{2}$ and $\theta_{3}$, and its mathematical model 
Red line in Fig.4 shows the approximated curve by the mathematical model. As shown in the figure, each of the coefficient of determination $R^{2}$ becomes 0.9953 and 0.9965 , and the model can reproduce human characteristics accurately.

In this research, a new mechanism which has two pairs of curve-shaped piston and cylinder mounted at the outside and the inside of the leg is proposed. This mechanism can passively follow the curves shown in Fig. 3 and Fig. 4 when the leg moves from bending position to extension position.

\section{Proposal of the orthotic device in consideration of the screw home movement}

\subsection{Mechanism of the proposed orthotic device}

A new orthotic device which places a curve-shaped piston and cylinder in the outside and the inside of the leg is proposed as shown in Fig.5. Here, the device shown in the figure is for the right foot. In the device, the cylinder is arranged to the upper leg, and the piston is arranged to the lower thigh. The cylinder and the piston on the right in the figure is arranged at the inside of the leg, and the cylinder and the piston on the left in the figure is arranged at the outside of the leg.

Valgus rotation is realized by giving different curvature of the cylinder and the piston at the inside with that at the outside in the $X$ - $Y$ plane. SHM is realized by giving different curvature of the cylinder and the piston at the inside with that at the outside in the $Y-Z$ plane.

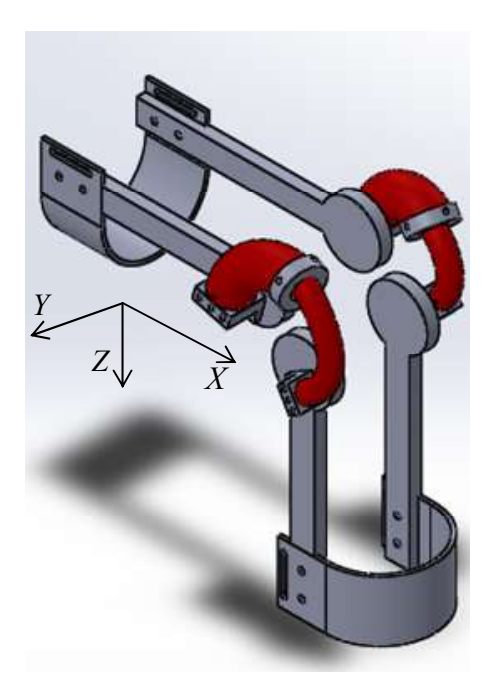

Fig.5 Proposed orthotic device

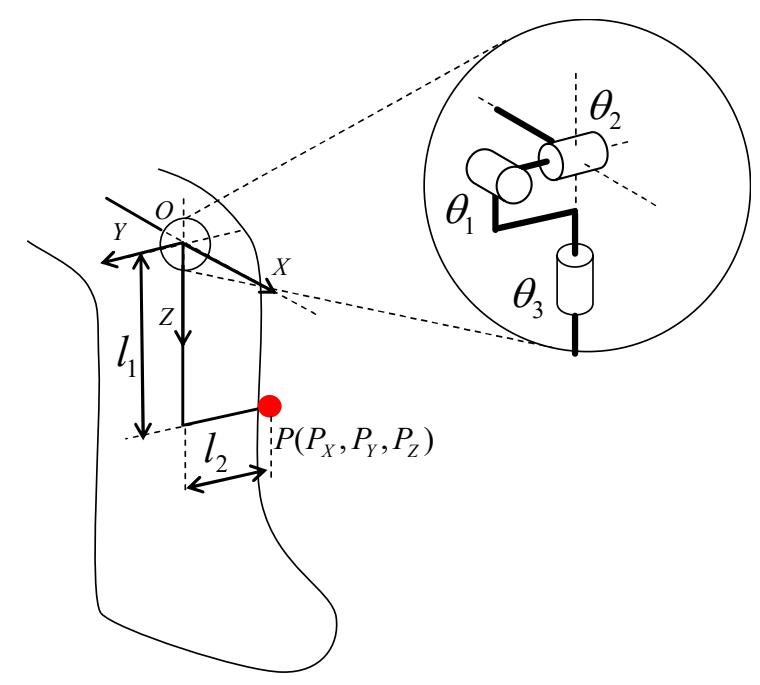

Fig.6 Link coordination system

\subsection{Derivation of the shape of the support part for the inside of the lower thigh}

As suggested in the previous section, the support part for the inside of the lower thigh is designed to rectify the movement of the lower thigh of patients to follow valgus rotation and SHM of healthy human. Therefore the curvature of inside cylinder and piston is estimated by using the Denavit-Hartenberg method (DH method) based on the model described in equation (1) and (2). A link coordinate system shown in Fig.6 is defined to use the DH method for the movement of knee joint. Where, $O$ is the origin, which is located at the center of knee joint, and $P\left(P_{X}, P_{Y}, P_{Z}\right)$ is the representative position on the curvature. $l_{1}$ is the distance between $P$ and the origin in $Z$ axis when $\theta_{2}$ is 90 degree. $l_{2}$ is the distance between $P$ and the origin in $Y$ axis when $\theta_{2}$ is 90 degree. $l_{1}$ and $l_{2}$ are determined as 50 mm considering width and thickness of the lower thigh.

Trajectory of the point $P$ calculated by the DH method is shown in Fig.7, which can be approximated by an arc of a radius of $228.35 \mathrm{~mm}$. In addition, trajectory of the point $P$ in the $X-Z$ plane can be approximated by an arc of a radius of $49.31 \mathrm{~mm}$ as shown in Fig. 8 . 


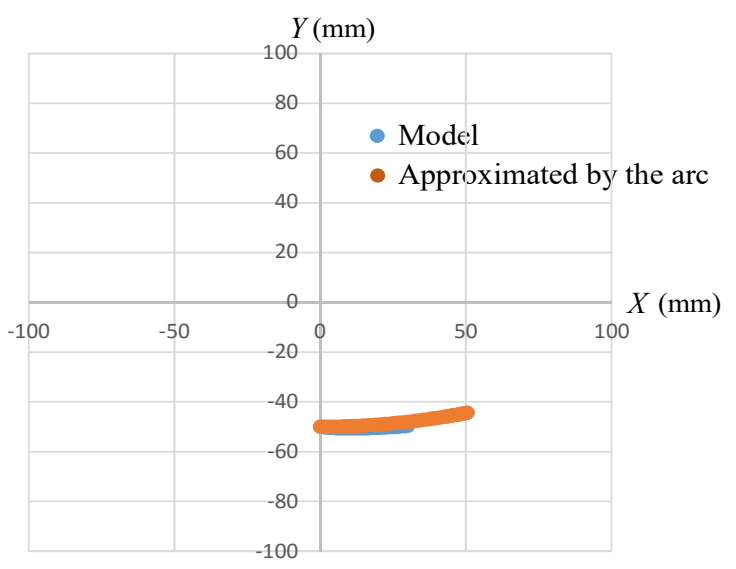

Fig.7 Position of $P$ and its approximated value $(X-Y$ axis $)$

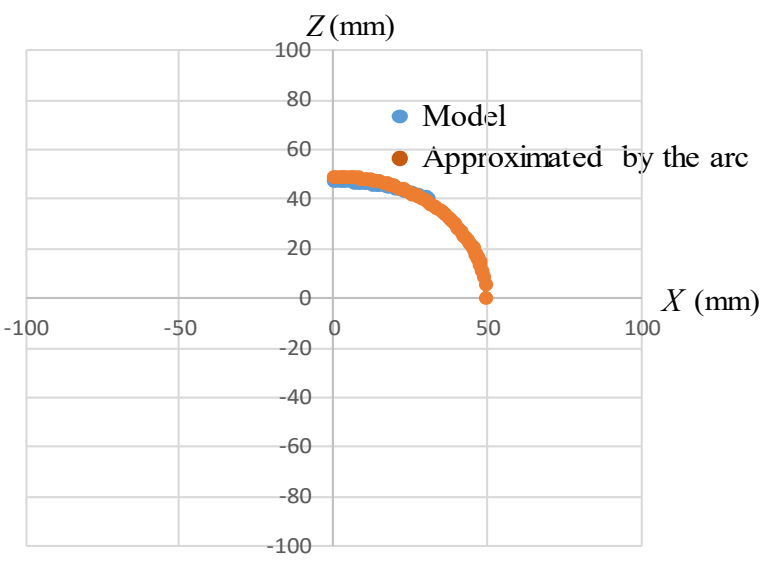

Fig.8 Position of $P$ and approximated value $(X-Z$ axis $)$

\subsection{Design of the orthotic device}

The shape of the cylinder and the piston for the outside of the lower thigh is decided based on the shape of the cylinder and the piston for the inside of the lower thigh. First, the curve of the outer portion is necessary to follow the inside curve in consideration of the width of the legs. Here, leg width is set to $80 \mathrm{~mm}$ in reference to the mean of the subjects. In addition to this, considering the structure of the cylinder (cylindrical diameter 20mm), offset value of the outer portion is decided to $110 \mathrm{~mm}$. In other words, curvature radius of the outer support part in $X-Y$ plane is $110 \mathrm{~mm}$ smaller radius than that of inner arc as shown in Fig.9. Here, the central angle of the inner arc is $12.5 \mathrm{deg}$ when the value of $X$ becomes 49.31 so that $Z=0$ in $X-Z$ plane. The central angle of the outside of the arc is also the same value of $12.5 \mathrm{deg}$. The radius of the inside arc in $X-Z$ plane is set to $49.31 \mathrm{~mm}$. The radius of the outer arc in $X-Z$ plane is determined to be the value of $X$ when the central angle of the outer arc is $12.5 \mathrm{deg}(X=25.62 \mathrm{~mm})$.

The piston and cylinder are formed along the inner and outer arc resulting from the above shown in Fig.9.

The size of the device is referenced to that of the commercially available knee brace. The size of each part is shown in Fig.10. The length from the rotation center to the end of stay in Fig.10(a) is 220mm in bending posture. An anchor is attached at the end of the stay. The device is mounted to human leg by two pairs of anchor and strap at both the femur side and lower thigh side. Fig.10(b) shows the extending posture of the knee joint. It is found that the device can carry out $8.1 \mathrm{deg}$ valgus rotation and 9.0deg SHM after extending. It is expected that these movements can assist a normal rotation which reduces the pain of the OA patients. 


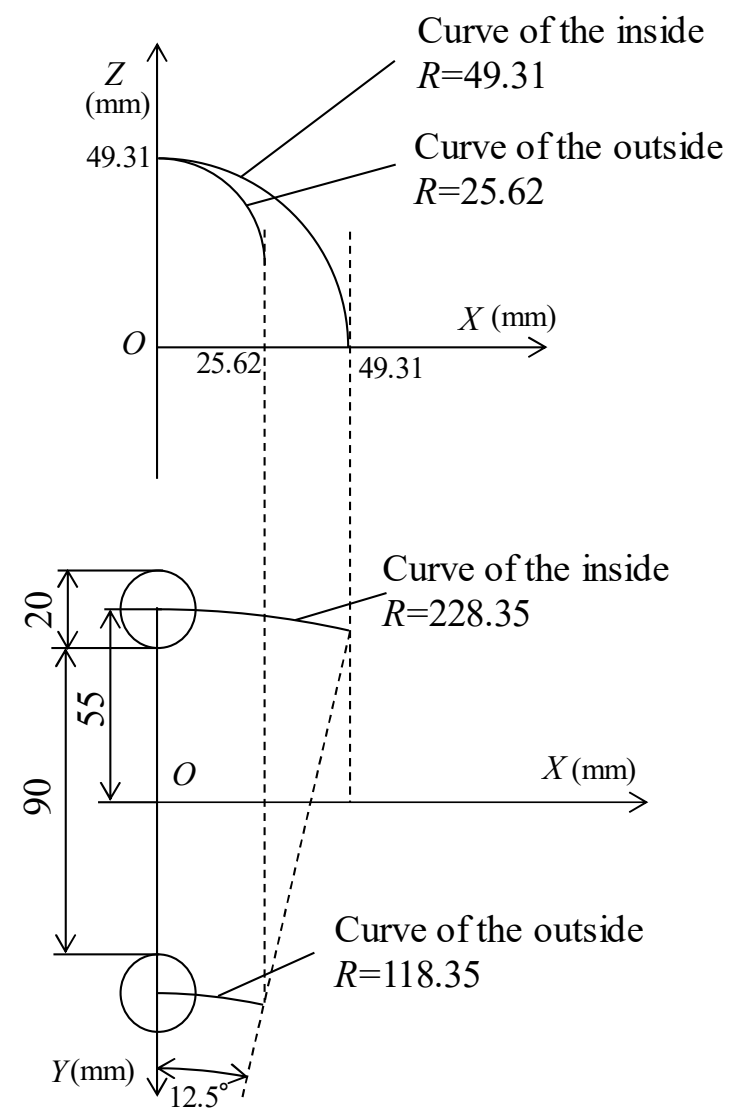

Fig.9 Determination of curvature radius

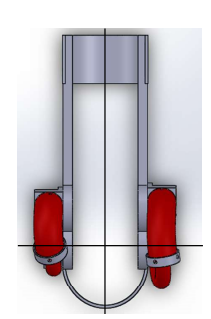

Top view

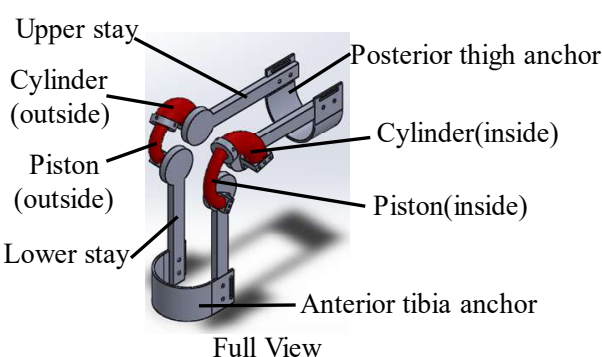

Full View

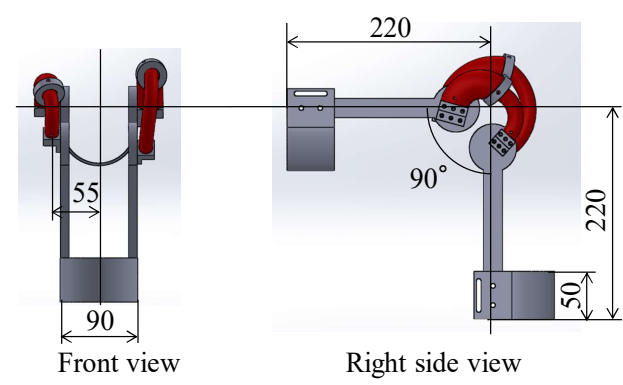

(a) When the knee joint is bending

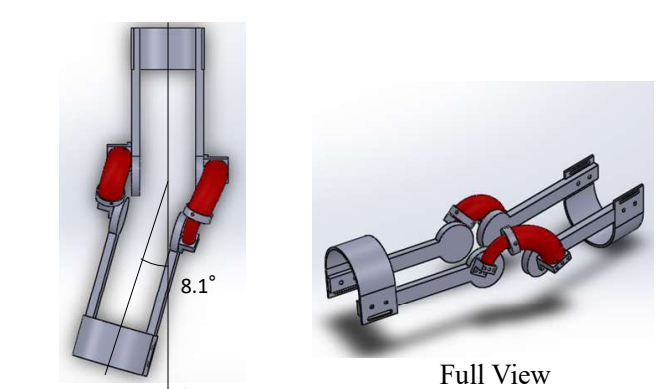

Top view

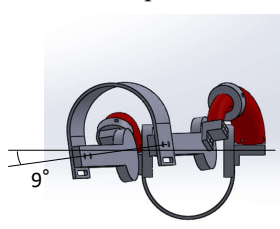

Front view

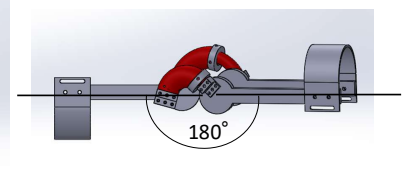

Right side view (b) When the knee joint is extending

Fig.10 Design of the orthotic device

\section{Validation of the effectiveness of orthotic device by FEM simulation}

\subsection{FEM model of orthotic device and leg}


To confirm qualitatively the mechanical effects of the orthotic device to the leg, a simple FEM model of the orthotic device and the right leg is created in MSC-NASTRAN, a well-known commercially available FEM software package. As shown in Fig.11, the orthotic device is being worn on the leg while the leg moves from bending position to extension position.

In this analysis, the sizes of a human leg and the device are decided from the average size of adult males. Hence the anchors at the top and bottom of the device contact the leg firmly. The bone column, which has a diameter of $3.0 \mathrm{~cm}$, is set at the center of the leg and surrounded by muscles and skin. The muscle and skin are treated as nonlinear material, their material properties are referred to web page of RIKEN (RIKEN, 2016).

The Young's modulus and Poisson's ratio of bone are $14 \mathrm{GPa}$ and 0.3 , respectively. The material of orthotic device is temporally assumed to be aluminum alloy. The Young's modulus and Poisson's ratio of it are 70GPa and 0.3, respectively. The top of the bond is fixed, and boundary condition between the device and the leg is assumed to be contact condition.

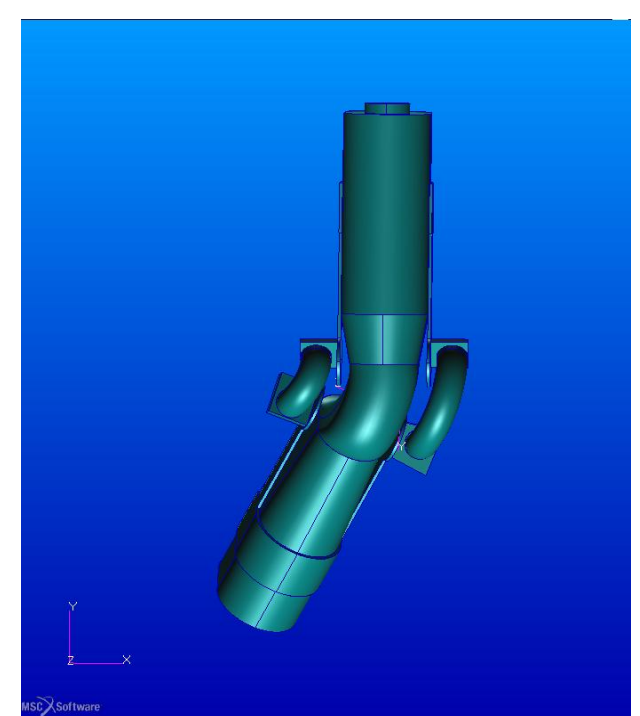

Fig.11 Model of orthotic device and right leg

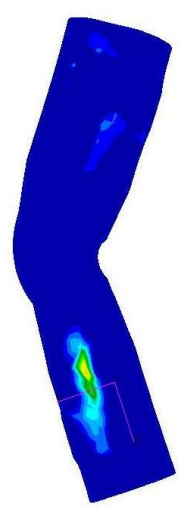

(a) Inside

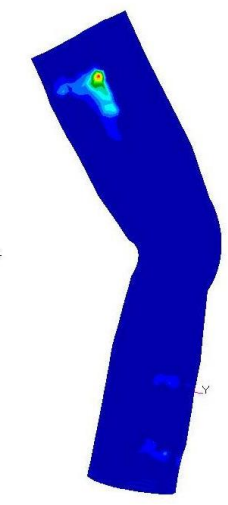

(b) Outside

$$
\times 10^{4} \mathrm{~Pa}
$$

0.00

Fig.12 Pressure of orthotic device acting on leg

\subsection{Analysis and result}

As stated in chapter3, the device can carry out 8.1deg valgus rotation and 9.0deg SHM of lower thigh when stretching knee from bending posture. The FEM analysis is performed according to these movements. Fig. 12 shows the pressure distribution applied to the leg when the leg moves from flexion position to extension position. It is found that the high pressure area mainly concentrates at the outside of the upper anchor position and at the inside of the lower let holder position. It is confirmed that the force of the device acting on the leg is helpful to realize SHM and valgus rotation.

\section{Conclusion}

In this study, as a fundamental approach to realize an orthotic device for patients with osteoarthritis of the knee considering screw home movement, extension movements of lower thigh from bending posture of able-bodied persons are analyzed firstly. As the results, valgus rotation and screw home movement are observed, and their characteristics are examined. The valgus rotation and screw home movement are mathematically modeled respectively, and it is confirmed that the models can reproduce their movements accurately.

Secondly we proposed the construction of the orthotic device using a cylinder and a piston. The shapes of the cylinder and the piston are designed based on the mathematical models to correct the extending motion of lower thigh 
of osteoarthritis patient.

Thirdly the force applied to human leg when using the proposed orthotic device is examined by FEM analysis, and it is confirmed that the force is helpful to realize normal valgus rotation and screw home movement, which shows the effectiveness of the proposed orthotic device.

\section{Acknowledgements}

This project is partly supported by the Grant-in-Aid for Scientific Research(C) No.26350660 from Japan Society for the Promotion of Science KAKENHI, Japan.

\section{References}

Esrafilian, A., Karimi, M.T. and Eshraghi, A., Design and evaluation of a new type of knee orthosis to align the mediolateral angle of the knee joint with osteoarthritis, Advances in Orthopedics, (2012), Article ID 104927.

Goldberg, V. M., Surgery in osteoarthritis: general conditions, Osteoarthritis; Diagnosis and Medical/Surgical Management, 2nd edition(1992), pp.535-543.

Matsuno, H., Kadowaki, K. M. and Tsuji, H., Generation II knee bracing for severe medical compartment osteoarthritis of the knee, Archives of Physical Medicine and Rehabilitation, Vol.78, (1997), pp.745-749.

Pollo, F. E., Otis, J. C., Wickiewicz, T. L., Warren, R. F. Biomechanical analysis of valgus bracing for the osteoarthritic knee, Clinical Gait Laboratory Conference, (1994)

Postelmans, R.J.J.,Knee support or replacement apparatus, US patent disclosure 5611774 A(1997).

RIKEN, Human organs property database for computer simulation (online), available from $<$ http://cfd-duo.riken.jp/cbms-mp/>, (accessed on 31 March, 2016).

Sasaki, T. and Yasuda, K., Clinical evaluation of the treatment of osteoarthritic knees using a newly designed wedge insole, Clin Orthop, Vol.221, (1987), pp.181-187.

Self, B. P., Greenwald, M. R. and Pflaster, D. S., A biomechanical analysis of a medial unloading brace for osteoarthritis in the knee, Arthritis Care and Research, Vol.13, No.4 (2000), pp.191-195.

Wu, Z. Q., Takahashi, M., Motokubo, Y., Sogabe, Y., Okada, T., Yamasaki, J., Experiment and Shape Design of Frame of New Type OA Knee Supporter, Proceedings of the SEM XIth International Congress and Exposition on Experimental and Applied Mechanics, (2008)

Yamasaki, J. and Moriwaki, T., Knee brace, US patent disclosure 6953442 B2 (2005).

Yasuda, K. and Sasaki, T., The mechanics of treatment of the osteoarthritic knees with a wedged insole, Clin Orthop, Vol.215, (1987), pp.162-172. 